\title{
Quasiregular quantum-dot-like structure formation with postgrowth thermal annealing of InGaN/GaN quantum wells
}

\author{
Yen-Sheng Lin, Kung-Jen Ma, and Cheng Hsu \\ Department of Mechanical Engineering, Chung Cheng Institute of Technology, National Defense University, \\ Tahsi, Taoyuan, Taiwan, Republic of China \\ Yi-Yin Chung, Chih-Wen Liu, Shih-Wei Feng, Yung-Chen Cheng, C. C. Yang, ${ }^{\text {a) }}$ \\ and Ming-Hua Mao \\ Department of Electrical Engineering, Graduate Institute of Electro-Optical Engineering, and Graduate \\ Institute of Electronics Engineering, National Taiwan University, I, Roosevelt Road, Section 4, \\ Taipei, Taiwan, Republic of China
}

Hui-Wen Chuang, Cheng-Ta Kuo, and Jian-Shihn Tsang

Advanced Epitaxy Technology Inc., Hsinchu Industrial Park, Taiwan, Republic of China

Thomas E. Weirich

The Center of Electron Microscopy, Aachen University of Technology, Ahornstrasse 55, D-52074 Aachen, Germany

(Received 22 October 2001; accepted for publication 5 February 2002)

\begin{abstract}
Postgrowth thermal annealing of an InGaN/GaN quantum-well sample with a medium level of nominal indium content (19\%) was conducted. From the analyses of high-resolution transmission electron microscopy and energy filter transmission electron microscopy, it was found that thermal annealing at $900{ }^{\circ} \mathrm{C}$ led to a quasiregular quantum-dot-like structure. However, such a structure was destroyed when the annealing temperature was raised to $950{ }^{\circ} \mathrm{C}$. Temperature-dependent photoluminescence (PL) measurements showed quite consistent results. Blueshift of the PL peak position and narrowing of the PL spectral width after thermal annealing were observed. (C) 2002 American Institute of Physics. [DOI: 10.1063/1.1467983]
\end{abstract}

Quantum-dot-like structures have been observed in InGaN/GaN quantum wells (QWs). Such structures are formed because of the large lattice mismatch between $\mathrm{InN}$ and GaN. ${ }^{1-3}$ With high-resolution transmission electron microscopy (HRTEM), randomly distributed clusters of indium aggregation and phase-separated InN were widely observed. The cluster structures form potential minimums (called localized states) for trapping carriers for efficient photon emission. ${ }^{4-7}$ Such indium-aggregated distributions are basically located in the quantum-well layers; however, they usually diffuse into barriers, extensively under certain conditions. Typically, such out-diffusion becomes more extensive in a sample with a higher nominal indium content. ${ }^{3}$

It has been reported that postgrowth thermal annealing could alter the sizes and distributions of self-organized InAs quantum dots. $^{8,9}$ In InGaN compounds, postgrowth thermal annealing processes for changing cluster structures and their photon emission properties were also reported. ${ }^{10-12}$ In one of the previous results, a better-confined quantum-well structure, i.e., weaker indium out-diffusion, after thermal annealing was reported. ${ }^{12}$ The photoluminescence (PL) intensity was increased with the stronger quantum-well confinement effect. Postgrowth thermal annealing can provide means for device manufacturers to tune the photon emission wavelength or to tailor the gain spectrum by changing the size/ composition of the quantum-dot-like structures. The information of thermal annealing is also helpful for crystal

${ }^{a}$ Electronic mail: ccy@cc.ee.ntu.edu.tw growers to design growth procedures. In this letter, we report the formation of quasiregular quantum-dot-like structures from randomly distributed indium-aggregated clusters after postgrowth thermal annealing of an InGaN/GaN QW sample. It was found that with thermal annealing temperature at $900{ }^{\circ} \mathrm{C}$, indium distribution was better confined in the quantum-well layers. Although the better confinement in a high-indium sample $(>45 \%)$ has been reported, ${ }^{12}$ the observation of quasiregular quantum-dot-like structures has never been reported. In particular, the indium content of our sample is much lower $(19 \%)$. Also, we found that such a trend of regular structure disappeared when the thermal annealing temperature was $950^{\circ} \mathrm{C}$. The measurements of optical properties showed quite a consistent trend.

The sample used in this study was grown with a lowpressure metal-organic chemical-vapor deposition reactor. The InGaN/GaN QW sample consisted of ten periods of In$\mathrm{GaN}$ wells with $3.5 \mathrm{~nm}$ thickness. The barrier was $10 \mathrm{~nm}$ GaN. In the sample, the QW layers were sandwiched between a $1.5 \mu \mathrm{m}$ GaN buffer layer on a (0001) sapphire substrate and a $50 \mathrm{~nm}$ GaN cap layer. The growth temperatures were 1010 and $720{ }^{\circ} \mathrm{C}$ for $\mathrm{GaN}$ and $\mathrm{InGaN}$, respectively. The designated indium content of the sample was about $19 \%$. As-grown samples were thermally annealed in a quartz tube furnace at different temperatures ranging from 800 to $950{ }^{\circ} \mathrm{C}$ in nitrogen ambient for $30 \mathrm{~min}$. HRTEM and energy filter transmission electron microscopy (EFTEM) were used to characterize material structures.

For HRTEM observation, cross-sectional samples were prepared in the conventional manner by grinding, dimpling, 

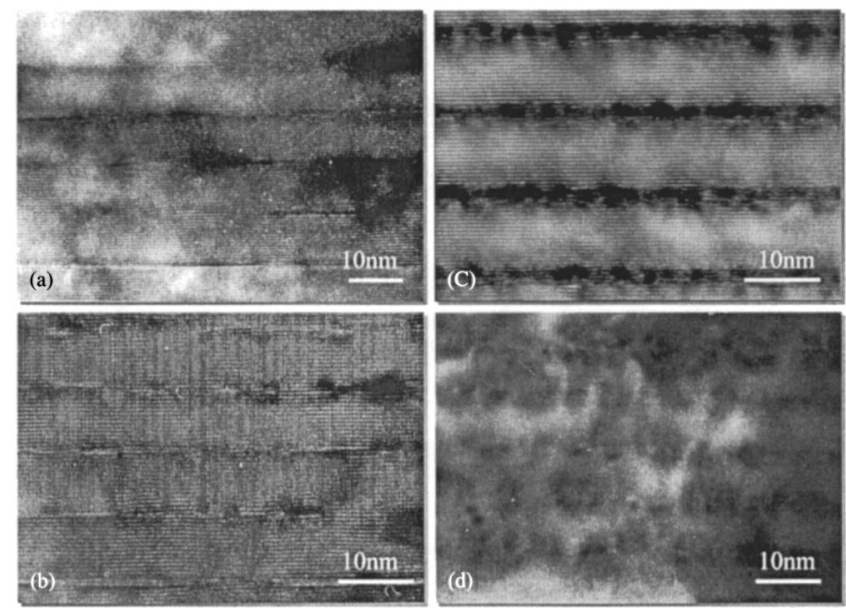

FIG. 1. HRTEM bright-field images of the as-grown (a), $800{ }^{\circ} \mathrm{C}$-annealed (b), $900{ }^{\circ} \mathrm{C}$-annealed (c), and $950^{\circ} \mathrm{C}$-annealed (d) samples.

and $\mathrm{Ar}^{+}$-ion milling with $6 \mathrm{kV}, 1 \mathrm{~mA}$, and an incident angle of $4^{\circ}$. Based on previous experiences, for samples containing indium, the ion-milling step needs to be carried out with the specimen holder at liquid-nitrogen temperature in order to minimize ion-beam damage. The HRTEM investigations were conducted with a $200 \mathrm{keV}$ Philips CM 200 and a 300 keV JEM 3010 microscope. All the high-resolution micrographs were taken at Scherzer defocus and the sample was viewed along a [11-20] zone axis. The $300 \mathrm{keV}$ JEM 3010 microscope was equipped with a $2 \mathrm{k} \times 2 \mathrm{k}$ slow-scan chargecoupled-device camera and a Gatan imaging filter (GIF). Regarding the EFTEM images, the drift between the two preedge and the postedge images was recorded by using the cross-correlation algorithm available in the Digital Micrograph software of the GIF. In order to remove the background contribution underneath the ionization edges and to obtain elemental maps, the jump-ratio method was used to avoid noise and artifacts. However, it cannot be quantified.

Figures 1(a)-1(d) show the HRTEM bright-field images of the as-grown (a), $800^{\circ} \mathrm{C}$-annealed (b), $900^{\circ} \mathrm{C}$-annealed (c), and $950^{\circ} \mathrm{C}$-annealed (d) samples. The contrast variations in the pictures represent the fluctuations of indium composition. The diffusive InGaN/GaN QW interfaces can be clearly seen in the as-grown sample [Fig. 1(a)]. The average size of indium-rich clusters is larger than $10 \mathrm{~nm}$. The clusters are irregularly dispersed and extended into the GaN barriers. Increasing the annealing temperature leads to a better confinement of indium-rich clusters near InGaN QWs, as shown in Figs. 1(b) and 1(c). The average size of the indium-rich clusters becomes smaller after the annealing treatment. The size homogeneity of quantum-dot-like structures was also improved. Interestingly, one can observe that very-fine indiumrich quantum-dot-like structures $(2-5 \mathrm{~nm})$ were regularly dispersed inside the InGaN QWs after the annealing treatment at $900{ }^{\circ} \mathrm{C}$ [Fig. 1(c)]. However, further increase of annealing temperature to $950^{\circ} \mathrm{C}$ leads to a highly irregular structure, as shown in Fig. 1(d). EFTEM scanning was performed for the samples. Indium composition profiles across and along the QWs were obtained. Line-scan results of indium composition (\%) across QWs in the as-grown sample (a), and samples annealed at $900{ }^{\circ} \mathrm{C}\left(\right.$ b) and $950{ }^{\circ} \mathrm{C}$ (c), are shown in Fig. 2. Compared to the as-grown sample, the

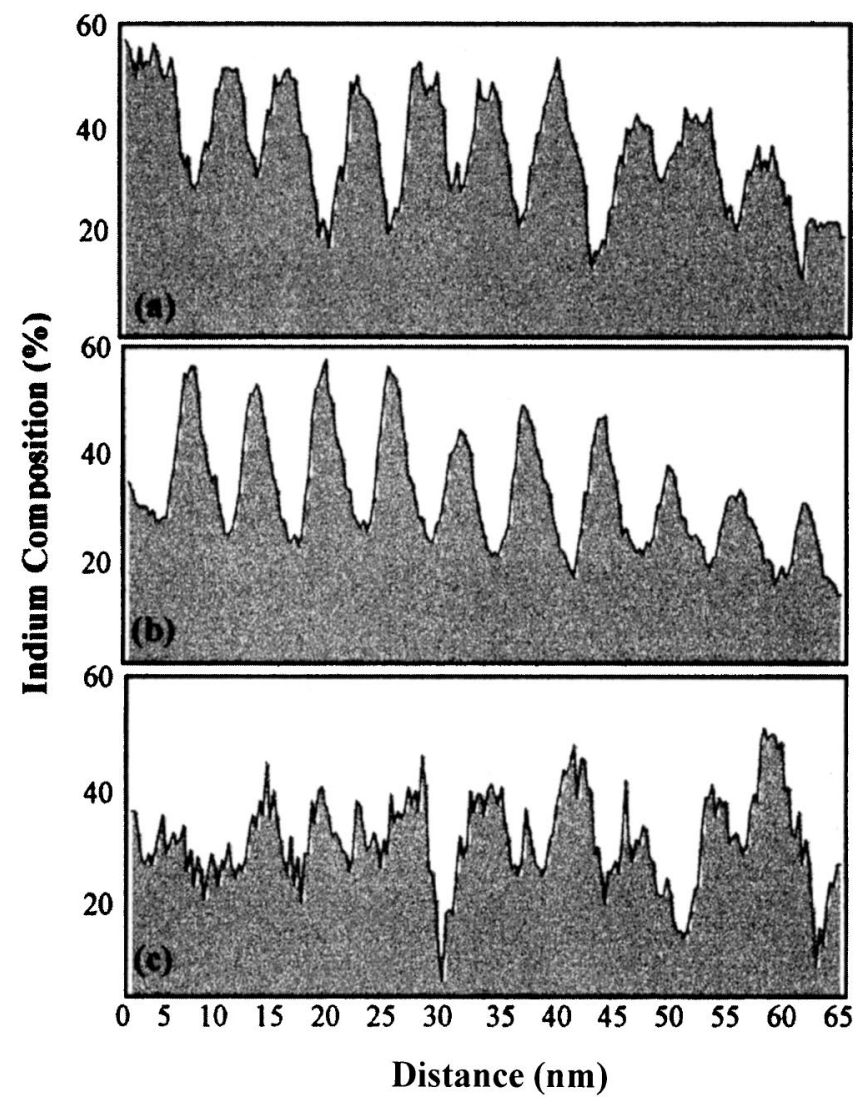

FIG. 2. EFTEM line-scan results of indium composition (\%) across QWs in the as-grown sample (a), and samples annealed at $900{ }^{\circ} \mathrm{C}(\mathrm{b})$ and $950{ }^{\circ} \mathrm{C}(\mathrm{c})$.

sample annealed at $900^{\circ} \mathrm{C}$ manifests better confinement of indium. However, after annealing at $950^{\circ} \mathrm{C}$ indium becomes dispersive again. Figure 3 shows EFTEM line-scan results of indium composition (\%) along a QW in the as-grown sample (a), and samples annealed at $850^{\circ} \mathrm{C}$ (b), $900{ }^{\circ} \mathrm{C}$ (c), and $950^{\circ} \mathrm{C}(\mathrm{d})$. Annealing did result in more regular structures of clusters. Quasiregularly arrayed quantum-dot-like structures with nearly the same indium concentration at the cores of the quantum-dot-like clusters can be observed in the sample with $900{ }^{\circ} \mathrm{C}$ annealing. The irregular distribution of compositional fluctuation can again be observed after postgrowth annealing at $950^{\circ} \mathrm{C}$. In the InGaN/GaN QW sample, thermal energy at $900{ }^{\circ} \mathrm{C}$ is sufficient to drive the system to a state of lower potential energy, i.e., a quasiregular structure. The relationship between the period of the indium-rich clusters and the width and nominal indium content of QWs is worth investigating further. When the annealing temperature reached $950^{\circ} \mathrm{C}$, the higher temperature caused coarsening of indiumrich clusters with the sizes exceeding the QW width. ${ }^{8}$ Such a process destroyed the QW structure, leading to the results shown in Figs. 1(d), 2(c), and 3(d).

Figures 4 and 5 show the PL characteristics of these samples. Because of the oscillatory variations in PL spectra due to the Fabry-Pérot effect, normal PL peak position and full width at half maximum could not be accurately calibrated. PL peak positions were then obtained through Gaussian fitting, and the root-mean-square (rms) spectral widths were calculated. Figure 4 shows the blueshifts of PL peak positions after thermal annealing. The usually observed S-shape variation of PL peak position is unclear in Fig. 4. 

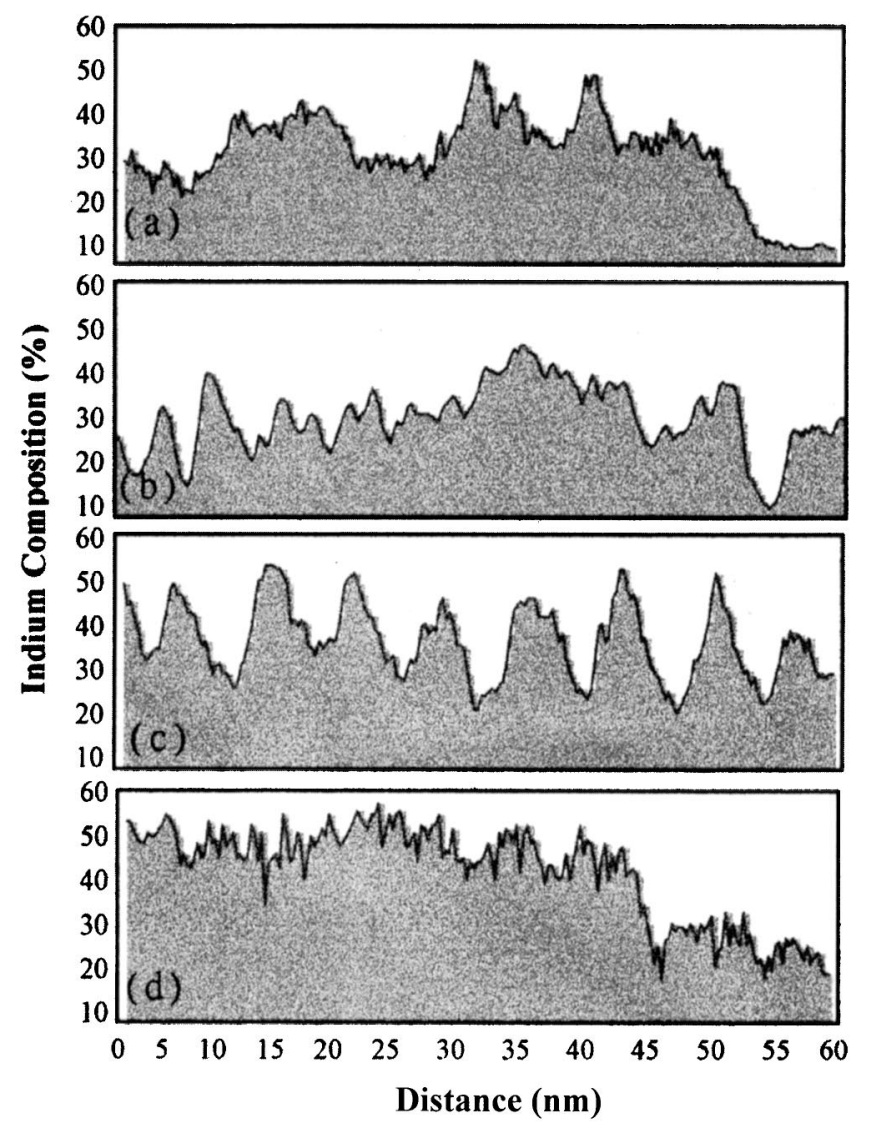

FIG. 3. EFTEM line-scan results of indium composition (\%) along a QW in the as-grown sample (a), and samples annealed at $850{ }^{\circ} \mathrm{C}(\mathrm{b}), 900{ }^{\circ} \mathrm{C}(\mathrm{c})$, and $950^{\circ} \mathrm{C}(\mathrm{d})$.

Typically, annealing at higher temperature results in a larger blueshift. However, the result of $950{ }^{\circ} \mathrm{C}$ annealing seems to have different behaviors between the low- and hightemperature ranges. Figure 5 shows the PL rms width variations of various samples. The as-grown sample shows an irregular variation in the high-temperature range. Typically, thermal annealing leads to more regular indium cluster structures. Therefore, the PL spectral width is expected to be

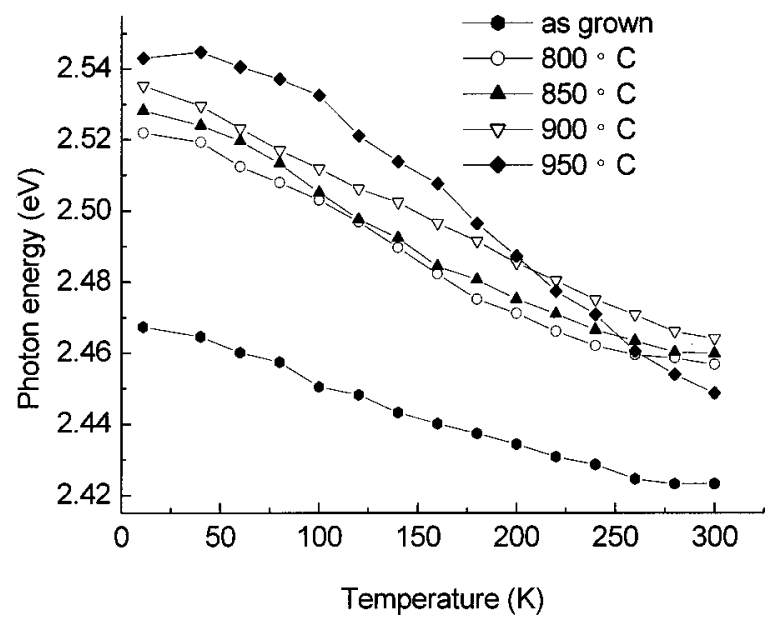

FIG. 4. Fitted PL peak positions as functions of temperature of various samples.

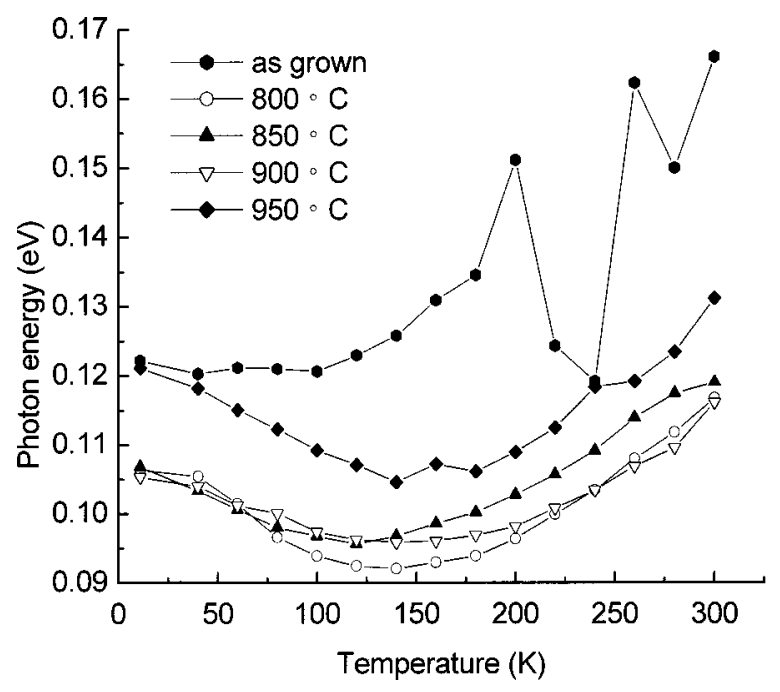

FIG. 5. PL root-mean-square spectral widths as functions of temperature of various samples.

smaller, as confirmed with the behaviors of the annealed samples in Fig. 5. The larger rms spectral width of the $950^{\circ} \mathrm{C}$ annealing sample, compared with the other three annealed samples, is consistent with the irregular structure shown in Figs. 1(d), 2(c), and 3(d).

In summary, we have conducted postgrowth thermal annealing of an InGaN/GaN QW sample with a medium level of nominal indium content. From material analyses, we found that thermal annealing at $900{ }^{\circ} \mathrm{C}$ led to quasiregular quantum-dot-like structure. However, such a structure was destroyed when the annealing temperature was further raised to $950{ }^{\circ} \mathrm{C}$.

This research was supported by the National Science Council, The Republic of China, under Grant Nos. NSC 892218-E-002-094, NSC 89-2218-E-002-095, and NSC 892215-E-002-051. This research was also supported by the Chung Shan Institute of Science and Technology, Taiwan, R.O.C.

\footnotetext{
${ }^{1}$ Y. Narukawa, Y. Kawakami, M. Funato, S. Fujita, S. Fujita, and S. Nakamura, Appl. Phys. Lett. 70, 981 (1997).

${ }^{2}$ H. Hirayama, S. Tanaka, P. Ramvall, and Y. Aoyagi, Appl. Phys. Lett. 72, 1736 (1998).

${ }^{3}$ Y. S. Lin, K. J. Ma, C. Hsu, S. W. Feng, Y. C. Cheng, C. C. Liao, C. C. Yang, C. C. Chuo, C. M. Lee, and J. I. Chyi, Appl. Phys. Lett. 77, 2988 (2000).

${ }^{4}$ S. Chichibu, T. Azuhata, T. Soda, and S. Nakamura, Appl. Phys. Lett. 69, 4188 (1996).

${ }^{5}$ S. Chichibu, T. Azuhata, T. Sota, and S. Nakamura, Appl. Phys. Lett. 70, 2822 (1997)

${ }^{6}$ M. S. Minsky, S. B. Fleischer, A. C. Abare, J. E. Bowers, E. L. Hu, S. Keller, and S. P. Denbaars, Appl. Phys. Lett. 72, 1066 (1998).

${ }^{7}$ Y. H. Cho, G. H. Gainer, A. J. Fischer, J. J. Song, S. Keller, U. K. Mishra, and S. P. DenBaars, Appl. Phys. Lett. 73, 1370 (1998).

${ }^{8}$ D. Bimberg, M. Grundmann, and N. N. Ledentsov, Quantum Dot Heterostructures (Wiley, Chichester, 1999).

${ }^{9}$ T. M. Hsu, Y. S. Lan, W. H. Chang, N. T. Yeh, and J. I. Chyi, Appl. Phys. Lett. 76, 691 (2000).

${ }^{10}$ W. H. Lee, K. S. Kim, G. M. Yang, C. H. Hong, K. Y. Lim, E. K. Suh, H. J. Lee, H. K. Cho, and J. Y. Lee, J. Korean Phys. Soc. 39, 136 (2001).

${ }^{11}$ L. T. Romano, M. D. McCluskey, B. S. Krusor, D. P. Bour, C. Chua, S. Brennan, and K. M. Yu, J. Cryst. Growth 189/190, 33 (1998).

${ }^{12}$ C. C. Chuo, C. M. Lee, T. E. Nee, and J. I. Chyi, Appl. Phys. Lett. 76, 3902 (2000).
} 
Applied Physics Letters is copyrighted by the American Institute of Physics (AIP). Redistribution of journal material is subject to the AIP online journal license and/or AIP copyright. For more information, see http:/ojps.aip.org/aplo/aplcr.jsp

Copyright of Applied Physics Letters is the property of American Institute of Physics and its content may not be copied or emailed to multiple sites or posted to a listserv without the copyright holder's express written permission. However, users may print, download, or email articles for individual use. 